Relations industrielles

Industrial Relations

\title{
Le "raisonnable" déraisonnable! ou la rationalité du raisonnable
}

\section{Fernand Morin}

Volume 40, numéro 3, 1985

URI : https://id.erudit.org/iderudit/050165ar

DOI : https://doi.org/10.7202/050165ar

Aller au sommaire du numéro

Éditeur(s)

Département des relations industrielles de l'Université Laval

ISSN

0034-379X (imprimé)

1703-8138 (numérique)

Découvrir la revue

Citer cet article

Morin, F. (1985). Le "raisonnable" déraisonnable! ou la rationalité du raisonnable. Relations industrielles / Industrial Relations, 40(3), 646-658.

https://doi.org/10.7202/050165ar
Résumé de l'article

Le « raisonnable deraisonnable! » ou la rationalite du raisonnable
Tous droits réservés (C) Département des relations industrielles de l'Universite Laval, 1985
Ce document est protégé par la loi sur le droit d'auteur. L'utilisation des services d'Érudit (y compris la reproduction) est assujettie à sa politique d'utilisation que vous pouvez consulter en ligne.

https://apropos.erudit.org/fr/usagers/politique-dutilisation/ 


\title{
Le «raisonnable» déraisonnable! ou la rationalité du raisonnable
}

\author{
Fernand Morin
}

Depuis quelques années, les multiples instances judiciaires, qui se croisent et s'entrecroisent en matière du travail, croient souvent nécessaire de marquer leurs interventions respectives du signe de la «raisonnabilité». Il suffit de parcourir les recueils de jurisprudence du Conseil canadien des relations du travail, des artibres de griefs, de la Cour supérieure, de la Cour d'appel, de la Cour fédérale et de la Cour suprême du Canada pour y retrouver d'innombrables variations sur le thème du raisonnable et du déraisonnable ${ }^{1}$. Ces deux mots, presque passe-partout, semblent parfois être utilisés comme s'il suffisait de les employer pour rendre raisonnable l'appréciation que l'on entend formuler ou pour déprécier inexorablement la décision infirmée. C'est alors que l'on voudrait savoir comment le caractère raisonnable d'une décision peut servir de critère déterminant pour l'exercice du contrôle judiciaire? Serait-il possible de s'assurer de la présence d'une «raisonnabilité» suffisante en une décision pour éviter ce contrôle? Comment répondre à ce type de question si on ne peut définir ce raisonnable ou à tout le moins, le distinguer du déraisonnable. Nous recherchons quelques éléments de réponses à ces questions tirées de l'enseignement de la Cour suprême du Canada donnés en deux décisions récentes.

Le 22 novembre 1984, la Cour suprême du Canada rendait deux jugements tendant a réduire l'usage de ce double qualificatif masquant trop de subjectivité et conduisant parfois à l'incohérence, au réel déraisonnable ou, à tout le moins, à l'insécurité juridique des parties ${ }^{2}$. Pour apprécier ces deux jugements, nous rappelons d'abord les questions soulevées, les décisions antérieures soumises à cet ultime contrôle judiciaire et l'approche finalement retenue par la Cour suprême du Canada. Nous formulons par la suite

* MORIN, F., professeur, Département des relations industrielles, Université Laval.

1 À titre d'illustration, nous rappelons que dans l'affaire Control Data que nous étudions ci-après, l'arbitre déclara devoir modifier la sanction imposée par l'employeur pour la rendre raisonnable, la Cour supérieure refusa l'évocation parce que la sentence n'était pas déraisonnable, la Cour d'appel considéra cette même sentence arbitrale tout à fait déraisonnable, alors que la Cour suprême du Canada infirma le jugement de la Cour d'appel parce que cette sentence n'était pas déraisonnable.

2 Syndicat des employés de production du Québec et de l'Acadie et C.C.R.T. et Société Radio-Canada, décision 16613, du 22 novembre 1984; Blanchard et Control Data Canada Ltée et $J .-P$. Lalancette, décision 17680 du 22 novembre 1984. 
quelques commentaires sur la portée de ces deux jugements et notamment, au sujet de l'usage du critère de la «raisonnabilité» pour les fins du contrôle judiciaire ${ }^{3}$.

\section{L'AFFAIRE SYNDICAT DES EMPLOYÉS DE PRODUCTION DU QUÉBEC ET DE L'ACADIE.}

Depuis quelques années déjà, l'obligation d'effectuer du surtemps faisait l'objet de griefs entre le syndicat et Radio-Canada. Selon un arbitre, la disposition conventionnelle laissait libre chaque salarié d'accepter le surtemps demandé alors qu'un autre arbitre fut, quelques années après, d'avis contraire. Au cours d'une convention collective, le syndicat décida que tous les salariés devaient refuser le surtemps demandé et c'est ce que ces derniers firent. L'employeur demanda au Conseil canadien des relations du travail (C.C.R.T.) de statuer sur la légalité de la grève et d'enjoindre le syndicat et les salariés à cesser un tel refus collectif. Se fondant sur les art. 180 et ss. du Code canadien du travail, le Conseil rendait, le 5 décembre 1979, une décision comportant notamment ces paragraphes 3 et 4:

«3. De plus, l'embargo sur le temps supplémentaire constitue une grève illégale au sens du Code et le Conseil le déclare.

Cependant, le Conseil a décidé, dans les circonstances présentes, et pour le moment, d'exercer sa discrétion de ne pas faire émaner d'ordonnance à ce sujet en ce qui concerne les employés de la Société à Montréal, mais il ordonne par les présentes que ledit embargo cesse immédiatement à Moncton et à Québec et que tous les employés membres de l'unité de négociation et que le syndicat intimé dans ces deux localité doivent se conformer à cette ordonnance immédiatement de même que le syndicat intimé qui devra en informer tous ses membres immédiatement.

4. Il est ordonné aux deux parties, le syndicat intimé et la Société Radio-Canada, Division française, de soumettre immédiatement le problème à savoir si le travail supplémentaire est facultatif ou non, selon les dispositions de la convention collective en vigueur présentement à un arbitre nommé selon les dispositions de l'article 155(2)c) ou /et d) du Code canadien du travail, en utilisant un des griefs présentement en suspens et portant sur cette même question. Ledit arbitre devra traiter cet arbitrage en priorité et selon la méthode d'arbitrage accéléré et sa décision devrait résoudre ce problème jusqu'à la signature d'une convention collective supplantant la présente qui contiendrait des dispositions différentes à ce sujet.»

En appel, la Cour fédérale du Canada reconnut la compétence du C.C.R.T. à qualifier, comme il le fit, ce refus collectif (par. 3 de la décision du Conseil) et cassa le par. 4 de cette même décision enjoignant les parties à soumettre l'affaire à un arbitre de grief. Ce jugement fut porté en appel à la Cour suprême du Canada par le syndicat sur la partie visant ce même par. 3 et le C.C.R.T. en appela du jugement de la Cour fédérale au sujet du par. 4 de sa propre décision (l'arbitrage obligatoire du litige). Nous étudions distinctement ces deux points.

3 Sous l'angle du droit administratif, le professeur Gilles Pépin a également analysé ces deux mêmes décisions: Les erreurs juridictionnelles et intrajuridictionnelles devant la Cour suprême du Canada in Revue du Barreau, tome 45, no 1, 1985, p. 117 et ss. 
Le refus collectif du surtemps est-il une grève? (par. 3)

Selon le syndicat, le Conseil canadien aurait commis une erreur sur une question attributive de compétence. Si l'art. 182 du Code l'autorisait à déclarer légale ou illégale une grève, cette qualification en serait une néanmoins attributive de compétence: un refus collectif de faire du surtemps, par ailleurs volontaire, ne pourrait constituer une grève. De plus, le syndicat prétendait qu'une telle qualification de grève au sens de l'art. 107 C.C.T., serait «... à tout le moins une erreur de droit manifestement déraisonnable lui enlevant toute juridiction et qui donne ouverture à une révision judiciaire». (p. 10)

La Cour suprême refusa de scinder la question en deux points de manière à faire de la qualification du refus collectif une question préliminaire attributive de compétence:

«Par cette division arbitraire des pouvoirs du Conseil, que ne justifient ni le texte de l'article 182 du Code, ni les principes, le Syndicat importe artificiellement dans le débat cette notion d'erreur sur un élément préliminaire mais essentiel à l'exercice de la compétence que cette Cour a justement voulu éviter dans l'arrêt la Société des alcools du Nouveau-Brunswick.» (1979) 2 R.C.S. 277 «La question de savoir s'il y a grève n'est pas préliminaire; elle fait partie intégrante du problème principal que seul le Conseil est chargé de résoudre: y a-t-il grève illégale?» (...) «J'ajouterai que tant la question de l'existence d'une grève que celle de sa légalité appartiennent à ce genre de questions qui relèvent de l'expertise particulière du Conseil et qu'il n'y a aucune raison théorique ou pratique - bien au contraire - de les scinder en les plaçant sur des plans différents»). (p. 12)

De plus, le plus haut tribunal considéra que ce par. 3 de la décision du Conseil canadien n'était nullement déraisonnable:

«Pour conclure que l'embargo collectif sur le temps supplémentaire est une grève, le Conseil s'est appuyé sur une jurisprudence qu'il qualifie de constante et que la Cour d'appel fédérale qualifie d'abondante, selon laquelle le droit des employés de refuser individuellement de faire du temps supplémentaire, lorsque ce droit existe, n'empêche pas que l'on puisse considérer qu'il y a grève au sens du paragraphe 107(1) du Code, lorsque ce droit est exercé de façon collective, concertée et systématique par les employés». (p. 13)

On souligne également que le Conseil canadien rendit sa décision en retenant la thèse syndicale à savoir que le surtemps était volontaire comme l'avait reconnu alors un arbitre. Plus tard, soit en 1981, un autre arbitre décida le contraire à savoir que le surtemps était obligatoire.

\section{L'arbitrage obligatoire par voie d'ordonnance (par. 4)}

Le Conseil canadien des relations du travail prétentait détenir l'autorité nécessaire pour enjoindre les parties à soumettre à l'arbitrage leur litige relatif au surtemps. Au soutien de cette thèse, le Conseil canadien soutenait qu'il lui fallait rechercher une solution pratique tenant compte de la guérilla judiciaire entretenue depuis plus de 6 ans entre les parties au sujet de cette question. Une telle ordonnance rendue en vertu de l'art. 182 C.C.T. devait 
«... renfermer les dispositions que, eu égard aux circonstances, le Conseil juge indiquées; ...» (art. 183.1, al. a, C.C.T.). Aussi, le Conseil canadien prétendait disposer «outre ses pouvoirs généraux ...» (art. 121) «... de pouvoirs discrétionnaires qu'il exerce en vertu des articles 182, 183 et 183.1(1) de manière à façonner des remèdes appropriés aux circonstances qui le confrontent». (p. 18) Finalement, le Conseil soutenait, que s'il avait commis quelques erreurs, cela ne pouvait être sur une question attributive de compétence et que le paragraphe 4 de sa décision n'était pas déraisonnable ni ne résultait d'une interprétation manifestement déraisonnable. La Cour suprême du Canada répondit distinctement à chacun de ces arguments.

Au sujet de l'étendue des pouvoirs d'intervention corrective du Conseil canadien, le juge Beetz en précise ainsi l'objet et la finalité:

«Si aucune des dispositions particulières invoquées par le Conseil n'a, séparément, l'effet de lui conférer le pouvoir qu'il a exercé, je ne vois pas comment l'addition de ces dispositions pourrait produire ce résultat. Or il me paraît certain qu'aucune d'entre elles n'a la portée que lui attribue le Conseil et qui donnerait un fondement juridictionnel au paragraphe 4 de son ordonnance.» (...) «... le Conseil se voit conférer le pouvoir d'assortir de modalités ou de conditions les ordonnances prévues à l'article 182; on en trouve un exemple au paragraphe 3 de l'ordonnance du Conseil qui restreint à Moncton et à Québec l'ordre de cesser l'embargo sur le temps supplémentaire. Sans doute le Conseil peut-il tenter d'adapter par là aux circonstances de chaque cas les ordonnances qu'il rend en vertu de l'article 182, mais il ne peut pas, relativement à des grèves illégales, rendre des ordonnances autres que celles prévues à cet article, quelqu'utiles qu'elles puissent être ou lui paraître.» (p. 123) (...) «Ici, le législateur a non seulement précisé les pouvoirs principaux du Conseil à l'article 182, mais également ses pouvoirs incidents à l'article 183.1. Ces deux articles décrivent de façon exhaustive la compétence du Conseil en matière de grève illégale et en épuisent le contenu.» (p. 25) (...) «Le pouvoir que le Conseil revendique implicitement par le paragraphe 4 de son ordonnance est d'une nature tout à fait exceptionnelle. Il s'agit du pouvoir d'intervenir d'autorité dans la procédure des griefs et de commander aux parties de la mener à terme en la conduisant d'une certaine façon. Le Code porte, à l'article 155, que toute convention collective doit contenir une clause de règlement définitif, sans arrêt de travail, par voie d'arbitrage ou autrement, de tous les conflits d'interprétation ou d'application de la convention collective et prescrit lui-même une procédure d'arbitrage lorsque la convention ne contient pas une telle clause». (p. 26)

En somme, la Cour suprême du Canada établit que le Conseil canadien ne disposait pas de tel pouvoir d'imposer l'arbitrage pas plus qu'il n'en aurait pour autoriser un salarié à recourir directement à l'arbitrage et aux frais du syndicat comme il l'avait déjà décidé dans une autre affaire (Syndicat des camionneurs c. Massicotte (1982) 1 R.C.S. 710).

Quand à savoir si une telle erreur portait sur une question attributive de compétence, le Conseil fédéral prétendait qu'il devait bénéficier du doute comme l'avait déjà reconnu la Cour suprême: «Il est souvent très diffícile de déterminer ce qui constitue une question de compétence. Ầ mon avis, les tribunaux devraient éviter de qualifier trop rapidement un point de question de compétence, et ainsi de l'assujettir à un examen judiciaire plus étendu, lorsqu'il existe un doute à cet égard". (Société des alcools du NouveauBrunswick (1972) 2 R.C.S. 227 à p. 233) Le juge Beetz souligna qu'un doute devait subsister pour qu'il en soit ainsi et que ce n'était pas le cas en cette affaire: 
«Est erronée à mon avis la prétention selon laquelle seule peut constituer une erreur juridictionnelle celle qui porte sur la compétence initiale, ou compétence ratione materiae, ou compétence d'attribution d'un tribunal administratif qui ouvre une enquête.» (...)

Il me paraît que si l'erreur juridictionnelle comprend celle qui porte sur la compétence initiale d'un tribunal administratif qui ouvre une enquête et sur son pouvoir de trancher par voie de déclaration la question qui lui est soumise, a fortiori s'étend-elle aux dispositions qui lui attribuent le pouvoir d'ajouter à sa décision finale des ordonnances destinées à donner suite à son enquête et à rendre ses déclarations efficaces par des injonctions mandatoires et autres mesures de redressement comme celles des alinéas a) à d) de l'article 182 . Je ne vois pas en vertu de quelle logique on limiterait à l'étape initiale la possibilité d'erreur juridictionnelle d'un tribunal administratif si ce dernier pouvait errer et excéder impunément sa compétence à l'étape de la conclusion qui constitue l'aboutissement de son enquête et son but ultime.» (p. 34) (...)

«Les dispositions que le Conseil devait interpréter en l'espèce sont attributives de compétence puisqu'elles portent sur les ordonnances que le Conseil est habilité à joindre à une déclaration de grève illégale. Il s'agit en effet de décider si le Conseil a le pouvoir de joindre à une telle déclaration une ordonnance de renvoi à l'arbitrage. Il me paraît donc non pas douteux mais manifeste que l'interprétation de ces dispositions soulève une question de compétence à propos de laquelle le Conseil ne peut errer sans commettre d'excès de compétence. Or, pour les raisons susdites, le Conseil a erré selon moi dans l'interprétation de ces dispositions et a exercé un pouvoir qui n'entre pas dans le cadre de ses attributions»). (p. 36)

Au sujet du troisième argument du Conseil canadien à savoir que le paragraphe 4 n'était pas déraisonnable, ni ne résultait d'une interprétation déraisonnable mais au contraire, qu'il s'agissait d'une ordonnance (l'arbitrage) qui tenait compte de toutes les circonstances, la Cour suprême du Canada reconnut qu'un tel moyen pouvait objectivement être justifié et être adapté à la situation de fait:

«Je veux bien admettre également cette autre proposition. Mais, à mon avis, elle n'est pas pertinente.

On aura observé qu'en l'espèce, si la Cour d'appel fédérale utilise le critère de l'erreur manifestement déraisonnable à propos de cette partie de l'ordonnance rendue par le Conseil qui entre dans le cadre de ses attributions, savoir, le paragraphe 3, elle ne souffle mot du même critère relativement au paragraphe 4 qui résulte d'une erreur juridictionnelle». (p. 37)

Puis, le juge Beetz apporte quelques précisions au sujet de ces qualificatifs de «raisonnable» ou de «déraisonnable»:

- Lorsqu'un tribunal de contrôle qualifie une décision de «non manifestement déraisonnable» cela ne signifierait pas que l'interprétation retenue est bonne ou mauvaise, mais bien que cette interprétation «peut se défendre». (p. 38) Ce tribunal de contrôle n'a pas à statuer sur le fond;

- Si la question soumise au contrôle en est une de compétence, le tribunal de contrôle doit établir si la décision visée est correcte ou non et il doit «(statuer sur l'exactitude de cette décision»... (p. 39);

- Le critère de l'interprétation «manifestement déraisonnable» ne s'appliquerait que dans les cas où la décision porte sur une question qui relève 
bien de la compétence de l'organisme. Si l'erreur touche une question relative à la compétence, il n'importe pas qu'elle soit manifestement déraisonnable, il suffit qu'il y ait erreur. (Banque Nationale du Canada (1984) 9 D.L.R. (éd. 10) Un tel contrôle des décisions des tribunaux administratifs, tout comme celui exercé à l'égard de la constitutionnalité des lois, résulterait de la même logique:

«Le pouvoir de contrôle des cours de justice a, dans les deux cas, le même fondement historique, et dans les deux cas il se rattache aux mêmes principes, la suprématie de la Constitution ou de la loi dans les cours de justice sont les gardiennes». (p. 43)

\section{L'AFFAIRE CONTROL DATA CANADA}

Suite à un congédiement, l'arbitre nommé en vertu de l'art. 124 de la Loi sur les normes du travail (L.N.T.) modifia la sanction en une suspension de quatre (4) mois. Contrairement à l'approche retenue par la Cour supérieure, la Cour d'appel (1983 CA 129) autorisa l'émission d'un bref d'évocation sous ce double chef: l'arbitre aurait rendu une décision déraisonnable en ces circonstances, bien qu'il soit nanti d'un tel pouvoir de correction (art. 128 L.N.T.) et la sentence arbitrale ne serait pas régulièrement motivée. La Cour suprême du Canada infirma ce jugement de la Cour d'appel. Trois questions furent soulevées devant cette haute instance:

1re question: L'existence d'une cause juste et suffisante de congédiement est-elle une condition préalable à l'exercice de la compétence de l'arbitre?

2e question: Compte tenu des pouvoirs qui lui sont conférés par l'article 128 L.N.T., l'arbitre a-t-il excédé sa juridiction en rendant une sentence déraisonnable?

3e question: Compte tenu de l'obligation de motiver la sentence arbitrale énoncée à l'art. 129 L.N.T., cette cour doit-elle intervenir et déclarer la sentence nulle faute de motivation suffisante?

Les notes de Messieurs les juges Lamer et Beetz apportent quelques précisions fort intéressantes au sujet de ces trois questions et que nous voulons rappeler, avant de commenter.

M. le juge Lamer cite un extrait du jugement de la Cour supérieure où on avait refusé l'évocation, notamment parce que: «On peut prétendre que sa sentence est trop sévère, on peut prétendre qu'elle n'est pas assez sévère, mais c'est lui seul l'arbitre qui est maître de sa sévérité. Il est là pour cela et s'il apparaît à la Cour que sa sentence est trop sévère ou pas assez sévère, la Cour ne peut permettre une évocation dans le seul but de la réviser dans un sens ou dans l'autre et de lui substituer son propre concept de sévérité». On rapporte également cet extrait des notes du juge Monet de la Cour d'appel alors dissident:

«Dans le cadre de la loi qui nous intéresse, l'arbitre a des pouvoirs autonomes. C'est d'ailleurs dans l'exercice de ces pouvoirs qu'il a décidé de ne pas annuler purement et simplement le congédiement. De la même façon, il a jugé équitable de substituer une 
mesure disciplinaire non draconienne, c'est-à-dire celle qui, compte tenu de son appréciation de l'ensemble de la preuve, était selon lui juste et raisonnable. Il n'a pas statué sur la légalité du congédiement mais sur la question de savoir si le mis en cause a raison de considérer qu'il a été traité injustement en étant congédié. N'est-ce pas là son rôle, tel que le veut la loi?» (1983) CA 129 à p. 142)

Outre cette «réhabilitation», M. le juge Lamer indique sous quelle approche les tribunaux devraient exercer leur fonction de contrôle:

«Elle ne doivent intervenir que si elles trouvent un véritable excès de juridiction de la part de l'arbitre et non simplement se trouvent en désaccord avec ses conclusions. Le contrôle judiciaire ne peut s'exercer ici que sur des questions de compétence». (p. 16)

Le juge Lamer fait sienne l'approche du juge Dickson qui était dissident dans l'affaire Jacmain:

«... à mon avis, les cours de justice devraient hésiter à déclarer un tribunal incompétent quand sa décision est honnête et équitable et qu'il a correctement pris en considération la documentation qui lui a été soumise. Dans l'exercice de son contrôle sur les conclusions en matière de compétence, la Cour doit laisser place à une certaine latitude. Elle doit se demander si la preuve est suffisante pour étayer les conclusions de fait et si les conclusions de droit ou les conclusions mixtes de fait et de droit sont logiques. L'erreur doit être manifeste. La Cour a un rôle de révision; elle ne doit pas faire un nouveau procès». (1978) 2 R.C.S. 15 à p. 19

Autrement, nous dit le juge Lamer, si un tribunal administratif ne pouvait se tromper sur toute question qualifiée de préliminaire, il pourrait difficilement exercer directement et exclusivement sa compétence propre:

«Dès lors, si toutes les conditions $\mathrm{X}$ sont dites préliminaires, le tribunal administratif a perdu la faculté de se tromper: il ne peut exercer le pouvoir qui lui est conféré par la loi que s'il a raison quant à son interprétation de ce que $\mathrm{X}^{1}, \mathrm{X}^{2}$ et $\mathrm{X}^{3}$ veulent dire. $\mathrm{Au}$ bout du compte, la distinction entre l'appel et le contrôle judiciaire est assez mince». (p. 20)

En cette affaire, le juge Lamer refuse de scinder en deux parties la démarche de l'arbitre pour considérer, à titre préliminaire, s'il existe une juste et suffisante cause:

«... il me semble clair que l'existence d'une cause juste et suffisante n'est pas une condition préliminaire a l'exercice de la compétence de l'arbitre. Il s'agit au contraire de l'objet même de l'enquête. C'est la seule question que l'arbitre doit décider avant de rendre l'ordonnance qu'il estime appropriée. La loi ne confère pas un pouvoir réparateur à l'arbitre dans l'abstrait: ce pouvoir lui est conféré lorsqu'il prend connaissance d'une situation qui mérite intervention, soit le congédiement sans cause juste et suffisante». (p. 22)

De même, la qualification des motifs de l'employeur relève-t-elle de la seule compétence de l'arbitre et s'il se trompe à ce sujet, il n'excèderait pas pour cela sa juridiction:

«... l'arbitre n'agit pas sans juridiction quand il substitue une nouvelle sanction à celle choisie par l'employeur après avoir erronément constaté l'absence de cause juste et suffisante de congédiement: il erre dans l'exercice de sa juridiction.» (...) 
«L'article 128 ne rend pas l'usage de ces pouvoirs conditionnel à l'existence objective de cette cause mais plutôt conditionnel à l'appréciation subjective de l'arbitre». (p. 23)

Existe-t-il des cas où l'erreur de droit commise par l'arbitre serait de telle nature qu'elle imposerait une intervention du tribunal? «... seules les erreurs de droit déraisonnables portent atteinte à la juridiction». (p. 24) Il en serait ainsi lorsque l'interprétation du tribunal administratif n'apparaît pas «... pouvoir rationnellement s'appuyer sur la législation pertinente». (C.U.P.E. à p. 237) Et le juge Lamer souligne qu'il s'agirait là d'un «... test très sévère et qui marque une approche restrictive en ce qui concerne le contrôle judiciaire». (p. 25) En somme, l'erreur doit être à ce point grave qu'elle porte atteinte à la juridiction et parce qu'elle est alors qualifiée de déraisonnable, il n'y aurait plus lieu de distinguer entre l'erreur de droit et celle de fait:

«L'erreur de fait déraisonnable a été qualifiée d'erreur de droit. La distinction voudrait qu'en un deuxième temps cette erreur de droit soit à l'abri de la clause privative à moins d'être déraisonnable, pour que, en devenant erreur de droit elle devienne une erreur de droit déraisonnable. Le tribunal administratif a la compétence voulue pour se tromper, et même gravement, mais n'a pas celle d'être déraisonnable. Ce qui est déraisonnable n'atrophie pas moins la juridiction du fait que la conclusion en est une de fait plutôt que de droit. La justification de l'intervention judiciaire est la conclusion déraisonnable». (p. 27)

En somme, lorsque le tribunal administratif est protégé par une clause dite privative, il ne serait plus nécessaire d'identifier les causes de l'erreur, ni de distinguer l'erreur de droit de l'erreur de fait et il suffirait que l'aboutissement, la conclusion du tribunal administratif soit déraisonnable:

«En conclusion, une détermination déraisonnable, quelle qu'en soit la source, porte atteinte à la juridiction du tribunal». (p. 28)

On saisit alors davantage le besoin de bien connaître quand et comment l'arbitre, en cette affaire Control Data, a pu déraisonnablement errer. D'abord, M. le juge Lamer rejette l'argument patronal voulant appliquer la décision Lafrance c. Commercial Photo Service Inc. (1980) 1 R.C.S. 536 pour soutenir que l'arbitre devait se limiter à établir s'il existe un motif sérieux au soutien de la sanction:

«... il faut donner un sens aux mots «juste et suffisante» dans l'art. 128 et qu'ils signifient qu'il doit exister une cause qui, selon l'arbitre, est suffisamment importante pour justifier un congédiement. En d'autres termes, il n'y a pas de cause juste et suffisante si, dans l'esprit de l'arbitre, le congédiement est une sanction disproportionnée par rapport à la faute». (p. 32)

L'arbitre a-t-il erré en imposant une sanction plus légère compte tenu de la gravité de la faute? Notons que c'est principalement sous cet angle que la Cour d'appel prit position et autorisa l'évocation. Le juge Lamer énonce d'abord le principe général qui lui servira de guide:

«La cour n'interviendra que si elle est satisfaite que l'arbitre a rendu une sentence déraisonnable. C'est une conclusion à laquelle les tribunaux ne devraient arriver qu'en se rappelant que l'arbitre est éminemment mieux placé pour juger de l'impact 
de la décision. En effet, faut-il encore le rappeler, les tribunaux administratifs répondent au besoin d'apporter des solutions à des conflits qui se prêtent mieux à un procédé décisionnel autre que celui qu'offrent les tribunaux judiciaires. Souvent aussi, le «juge» administratif est mieux formé et mieux renseigné sur le milieu où s'exerce sa compétence, et a accès à des renseignements qui ne se retrouvent pas, plus souvent qu'autrement, au dossier soumis à la cour. À cela s'ajoute le fait que l'arbitre a vu et entendu les parties». (p. 36)

Et il conclut ainsi:

«Cette Cour peut être d'accord ou pas avec la sentence arbitrale, cela ne l'autorise pas à substituer son opinion à celle d'un arbitre qui a agi conformément à sa loi habilitante et d'une façon qui n'est pas «manifestement déraisonnable». Or, il est clair que l'arbitre a tenu compte de toutes les circonstances entourant le congédiement et qu'il en a conclu que $\mathbf{M}$. Blanchard a été congédié pour diverses raisons, dont l'acceptation de «pots-de-vin» n'est qu'un élément. Il est concevable que dans la plupart des circonstances, une telle faute sera une cause juste et suffisante de congédiement. Toutefois, il n'a pas été démontré que, en regard des faits qu'a considérés l'arbitre et de sa position privilégiée, celui-ci a rendu une sentence déraisonnable en imposant une sanction moindre à l'appelant». (p. 37)

Au sujet de l'insuffisance de la motivation de la décision arbitrale, le juge Lamer applique la même logique:

«... il m'est difficile de voir comment un tel défaut dans les motifs pourrait porter atteinte à la juridiction de l'arbitre d'entendre le litige et de rendre la décision qu'il juge appropriée, sauf dans la mesure où l'insuffisance des motifs est tellement importante qu'elle équivaut à une violation des règles de justice naturelle». (p. 39)

M. le juge Beetz partage l'avis de son collègue Lamer et rappelle qu'il ne peut, «... guère ajouter aux motifs du juge Monet» (dissident à la Cour d'appel). Il ajoute cependant une observation d'un intérêt certain au sujet de ce reproche sévère formulé ainsi par la Cour d'appel:

«... interprétation déraisonnable des faits de l'affaire (il) a commis un excès de juridiction: sa décision manque totalement de réalisme et est contraire à l'ordre public... (elle) constitue un déni de justice flagrant, une incitation à la récidive et un mauvais exemple pour les autres employés». (1983) CA 129 à p. 134 et ajoute:

Le juge Beetz refuse d'y voir un abus de pouvoir de la part de l'arbitre

«La majorité en Cour d'appel me paraît avoir effectivement décidé que la seule sanction raisonnable du comportement certes répréhensible de l'appelant est nécessairement la sanction ultime, le congédiement, et qu'en imposant une sanction moins sévère, l'arbitre est allé à l'encontre de l'ordre public. 11 me semble, soit dit avec les plus grands égards, que c'est là venir près de confondre entre la conduite de l'appelant et celle de l'arbitre. Je suis loin d'être certain que j'aurais rendu la même décision que l'arbitre mais je suis également incapable d'affirmer que la pénalité moins sévère qu'il a imposée en remplacement de la pénalité ultime est, compte tenu de toutes les circonstances, clairement abusive, manifestement injuste, absurde, contraire au sens commun, et sans aucun fondement dans l'ensemble de la preuve». (p. 4) 


\section{COMMENTAIRES}

Ces variations de la Cour suprême du Canada sur le thème du «raisonnable» ne sont-elles pas révélatrices d'un certain malaise ou «... d'une rivalité à peine déguisée entre les cours traditionnels et les tribunaux...» administratifs ${ }^{4}$. On semble inciter les tribunaux à ne pas ériger en dogme ni en automatisme le critère du «raisonnable» et son antonyme. Ces deux derniers jugements s'inscrivent dans la démarche générale de la Cour suprême, «gardienne de la légalité», et qui consiste à assurer, à maintenir ou à restaurer au besoin l'harmonie, la cohérence, la complétude, l'efficacité ou, somme toute, la rationalité de notre système juridique. En ces deux occasion, a-telle atteint cet objectif? Depuis le 22 novembre 1984, pouvons-nous maintenant croire que l'on fera moins usage de ces mots passe-partout et de logique formelle pour s'en tenir à cet enseignement? Pouvons-nous espérer que dorénavant la Cour supérieure et la Cour d'appel n'interviendront que lorsque la conclusion du tribunal du travail ou celle de l'arbitre de griefs serait «... compte tenu de toutes les circonstances, clairement abusive, manifestement injuste, absurde, contraire au sens commun, et sans aucun fondement dans l'ensemble de la preuve»? (M. le juge Beetz ci-dessus)

Quelle leçon pouvons-nous tirer de ces deux jugements? Bien que la Cour suprême tente de cerner ce qui peut lui paraître raisonnable et qu'elle applique cette approche en ces deux affaires, il n'est pas surprenant qu'elle ne nous ait pas donné une définition objective, positive et valable en toute occasion pour reconnaître une décision raisonnable. Par les extraits déjà rappelés, on peut constater que l'on traite de la question par surimpression de petites touches, nuances et précisions, tantôt pour cerner le «raisonnable» et tantôt, pour accentuer les contrastes, en signalant ce qui serait «déraisonnable». La lecture de ces deux jugements fait voir d'ailleurs qu'il est peut-être plus commode d'utiliser la formulation négative, même très négative de "manifestement déraisonnable». L'emploi de ce double qualificatif indique d'autant plus la recherche de limiter l'usage de cette clef à l'évocation.

Selon ces deux jugements, la décision serait raisonnable si elle ne choque pas, si ses assises apparaissent fondées sur les dispositions législatives pertinentes et la jurisprudence applicable et aussi, si sa conclusion peut «se défendre», quelle que soit, par ailleurs, la position personnelle du juge qui exerce ce contrôle. On prend la peine de souligner, sans doute à l'intention des autres tribunaux, que le refus d'infirmer la décision du tribunal administratif, parce qu'elle n'apparaît pas manifestement déraisonnable, n'impliquerait nullement que l'on entérine sa conclusion. On ajoute même que l'arbitre doit demeurer seul «maître de sa sévérité» et qu'il procède nécessairement selon une «appréciation subjective des faits» et qu'il est possible que, dans l'exercice de sa compétence, il puisse se tromper même "gravement» sans pour cela rendre une décision déraisonnable. Par ailleurs, la décision de l'arbitre serait déraisonnable dès qu'il existe «... un véritable excès de juridiction». Il en serait ainsi si l'arbitre violait un principe fondamental de justice par exemple, l'absence réelle de motivation. Si la décision

4 Douglas S. Aircraft Company c. O'Shea \& all. (1980) 1 R.C.S. 245 d la p. 268, M. le juge Estey. 
sous contrôle porte sur une question attributive de compétence, il n'y aurait pas lieu de savoir si la conclusion est raisonnable ou si elle ne l'est pas ni de jauger la gravité de l'erreur. Si, en pareille occasion, il y a erreur, il s'agit d'un abus et la Cour suprême est d'avis que le tribunal de contrôle devrait y rémédier.

Outre ces premières précisions qui nous sont données en ces deux jugements, il importe de souligner l'approche générale de la Cour suprême:

- en ces deux cas, on incite les tribunaux à être circonspects et à n'intervenir que si le test sévère du «manifestement déraisonnable» les y autorisent;

- en ces deux mêmes cas, messieurs les juges Beetz et Lamer rappellent respectivement que les tribunaux administratifs (CCRT et l'arbitre) sont mieux placés pour apprécier l'ensemble des circonstances et que le législateur les a préférés à toute autre instance judiciaire pour trancher de telles affaires;

- dans l'affaire Control Data, le juge Lamer prit la peine de donner des extraits des deux jugements précédents (Cour supérieure et Cour d'appel) pour bien indiquer l'approche retenue et celle qui est manifestement rejetée. Le juge Beetz utilisa ce même moyen et ajoutait que la Cour d'appel s'était trompée de cible: elle avait frappé l'arbitre au lieu de l'employeur!

Ce n'est pas la première fois que la Cour suprême du Canada lance une telle slave d'avertissements aux autres tribunaux judiciaires. Plusieurs juges de la Cour suprême ont maintes fois invité ou incité la Cour supérieure et la Cour d'appel à modérer leurs interventions et matière arbitrales. Tout comme le syndrome de la mauvaise question qui consistait à alléguer que l'arbitre avait omis de répondre à la bonne question pour justifier l'évocation, le critère du déraisonnable pourrait être utilisé en fonction de la conclusion anticipée du tribunal de contrôle, c'est-à-dire qu'il entend infirmer la décision. À ce niveau, on ne peut guère y apporter quelque remède ${ }^{6}$. Par ailleurs, si ces mêmes tribunaux adoptaient l'approche générale proposée par la Cour suprême du Canada, le nombre de brefs d'évocation demandés et accordés, en première et deuxième instances, diminueraient sensiblement et, par effet d'entraînement, on peut croire que le nombre de requêtes décrô̂trait. Il s'agirait que la Cour supérieure et la Cour d'appel ainsi que les avocats en exercice acceptent la démarche générale retenue et soulignée à maintes reprises par la Cour suprême du Canada?.

5 À titre d'exemple, rappelons ces quelques décisions: Zeller's (Western) Limited c. Retail Wholesale and Department Store Union, local 955 \& al. (1975) R.C.S. 376; Bradburn \& al. c. Wenworth Arms Hotel Limited \& al. (1979) 1 R.C.S. 846; Douglas Aircraft Company of Canada Ltd c. O'Shea \& al. (1980) 1 R.C.S. 245; Volvo Canada Ltée c. Syndicat international des travailleurs unis de l'automobile \& al. (1980) I R.C.S. 178.

6 Comme en bien d'autres domaines, il faut chercher à convaincre, mais sans pouvoir réellement contraindre de suivre telle ou telle conduite: on peut offrir une nourriture, elle n'est pas pour cela consommée...

7 La Cour d'appel laissa entendre qu'elle avait reçu le message: $A$. Bastien c. Kraft Ltée, no 500-9-000003-348, Montréal, 15 février 85 et d'une manière encore plus certaine à Syndicat des employés municipaux de la Ville de Hull c. Ville de Hull décision du 3 sept. 85 no 500-9-000013-839 
Nous croyons qu'une décision ou conclusion d'un tribunal administratif ou arbitre pourrait être qualifiée de raisonnable ou, si l'on préfère, de non «manifestement déraisonnable» dès que l'on peut répondre positivement à ces questions:

- se situe-t-elle dans le cadre juridictionnel de l'organisme visé?

- la démarche retenue respecte-t-elle les principes fondamentaux de justice?

- résulte-t-elle de l'exercice des pouvoirs qui lui sont conférés par la loi tel que régulièrement reconnu par la jurisprudence?

- les motifs énoncés permettent-ils de constater que l'on a tenu compte de l'ensemble des circonstances de l'affaire peu importe la conclusion dégagée?

Au-delà de l'opinion personnelle du juge de contrôle, si ce dernier répond affirmativement à ces quatre sous-questions nous croyons que cette décision devrait paraître suffisamment cohérente et légitime pour être maintenue. C'est qu'alors cette décision s'inscrit dans la logique générale de notre système juridique comportant précisément cette répartition des tâches judiciaires entre les tribunaux réguliers et les organismes spécialisés ou ad hoc. Ce partage doit d'autant plus être respecté lorsque le législateur indique au surplus que les décisions des tribunaux administratifs ou spécialisés sont sans appel et dégagés de la supervision judiciaire (art. $139 \mathrm{Ct}$ ). Pareille décision devrait alors être qualifiée de raisonnable, parce qu'effectivement elle respecterait les qualités fondamentales recherchées dans un régime juridique: harmonie, cohérence et complétude des règles de droit et des instances du système judiciaire. Ces attributs ne sont-ils pas nécessaires pour atteindre ces trois objectifs de tout système juridique: stabilité, sécurité et prévisibilité?

À l'égard des rapports collectifs du travail, ces deux jugements apportent quelques précisions intéressantes au sujet de la définition de la grève et du rôle de l'arbitre de grief. Dans la décision concernant Radio-Canada, on confirme qu'une grève est bien un arrêt concerté de travail exercé à titre de moyens de pression auprès de l'employeur. Cette pression peut provenir de l'emploi d'un seul élément de la prestation de travail, en l'occurrence, le surtemps et même si, sur une base individuelle, cette prestation du surtemps n'était pas obligatoire. Un tel refus d'effectuer du surtemps changerait de nature lorsqu'il est collectif, lorsqu'il résulte d'une concertation et qu'il s'inscrit dans le processus des rapports collectifs du travail. À ces trois conditions, il s'agit d'une grève qui sera légale ou illégale selon que cet arrêt stratégique se réalise conformément aux conditions préalables à l'exercice du droit de grève. On voit que la Cour suprême du Canada s'est quelque peu écartée de la logique, peut-être trop simple, retenue dans une affaire de 1898 où des salariés s'étaient entendus pour refuser de travailler avec des nonsyndiqués. La Cour suprême avait alors fait ce bref raisonnement: «... les intimés dans l'occasion en question n'ont fait qu'user d'un droit qu'ils partagent avec leurs concitoyens de toute classe. Et ce droit, ils pouvaient s'entendre pour l'exercer tous ensemble, tout comme chacun d'eux pouvait le faire seul» ${ }^{8}$. Ce changement de nature juridique d'un pareil refus d'effec-

8 Perreault c. Gauthier (1898) 28 R.C.S. 214 à la p. 244. 
tuer du surtemps, selon qu'il est collectif ou individuel, ne peut nous surprendre parce que, dans l'un ou l'autre cas, l'arrêt ne produit nullement les mêmes effets et n'a pas la même signification:

- lorsqu'un salarié refuse le surtemps demandé, il le fait généralement pour des considérations personnelles et non pour exercer quelque pression sur l'employeur en vue de l'obtention d'un avantage;

- ce refus individuel ne perturbe pas le service car, d'ordinaire, d'autres collègues de travail acceptent le surtemps requis et ainsi, la charge de travail est effectuée selon le rythme et au moment désiré par l'entreprise;

- si ce refus est collectif et qu'il résulte d'une action concertée et stratégique, conçue et gérée par le syndicat, il est évident que la continuité du service en sera perturbée, dérangée sinon, arrêtée. La règle sous-jacente de la continuité du maintien des conditions générales de l'organisation de l'entreprise sont ainsi modifiées durant le terme d'une convention collective.

Dans l'affaire Control Data, la Cour suprême insiste sur deux caractéristiques de l'arbitrage des griefs: il s'agit du mode de justice choisi par le législateur au lieu et place des tribunaux ordinaires et deuxièmement, l'arbitre, ayant entendu les témoins et reçu directement tous les éléments mis en preuve, se trouve mieux placé que quiconque pour tenir compte «... de toutes les circonstances de l'affaire» (art. 100.12, al. f, Code du travail québécois et art. 156 C.C.T.). Le juge Lamer prit d'ailleurs la peine de souligner que l'arbitre avait effectivement tenu compte des circonstances particulières de l'affaire et du contexte.

La sentence de l'arbitre, tout comme le jugement de la Cour suprême indiquent d'ailleurs que ce congédiement s'inscrivait dans un contexte particulier et que l'on devait tenir compte de certains autres éléments et non seulement de la simple acceptation d'un voyage offert par un fournisseur de service. Non seulement la considération de toutes les circonstances de l'affaire permettait de comprendre la décision de l'arbitre, mais elle laisse, a contrario, comprendre que la Cour d'appel ne les considéra nullement, ce qui peut expliquer le ton vindicatif et la semonce qu'elle donna à l'arbitre.

Ces deux jugements de la Cour suprême du Canada soulèvent à nouveau la délicate et difficile question de l'usage modéré et pondéré d'un véritable et nécessaire moyen de contrôle des décisions abusives de l'arbitre et du tribunal du travail sans que ce procédé soit lui-même abusif. Il en va ainsi pour un meilleur respect du partage des attributions juridictionnelles édictées par la loi, pour une meilleure cohérence et stabilité du système judiciaire, pour la sécurité juridique des parties et enfin, pour une meilleure justice, allégée de ces artifices. On n'y arrivera pas par la voie de modifications à la loi, mais bien par celle de changements du comportement de tous (parties, avocats et juges). Ce n'est pas à l'occasion d'un dossier particulier que l'on peut se convaincre de la nécessité de tels changements d'attitudes, mais en considérant les méfaits de l'abus de ces moyens de contrôle sur la qualité générale des rapports collectifs du travail. Å suivre... 